\title{
A Fiber-Optic-Based 1550-nm Laser Radar Altimeter with RF Pulse Compression
}

\author{
Christopher Allen, Sivaprasad Gogineni \\ The University of Kansas, Radar Systems and Remote Sensing Laboratory \\ 2291 Irving Hill Road, Lawrence, Kansas 66045 USA \\ Telephone: 785/864-3017 Facsimile: 785/864-4971 email: callen@eecs.ukans.edu
}

\begin{abstract}
We are developing a laser radar (lidar) altimeter that uses commercial off-the-shelf fiber-optic components and traditional RF and digital signal processing techniques to achieve fine-range accuracy despite a low transmit peak power. A $1550-\mathrm{nm}$ operating wavelength was selected so that available erbium-doped fiber amplifiers (EDFAs) could be used to provide optical gain. The transmitted signal, an amplified, gated $\mathrm{CW}$ optical carrier intensity modulated with a linear-FM (chirp) RF signal, is launched into free-space via a fiber-optic collimator. An optical telescope serves as the receive aperture and couples the backscattered signal into a single-mode optical fiber prior to photodetection. The RF signal is then de-chirped and digitized for subsequent digital signal processing. Significant signal processing gains are achieved through coherent integration and pulse compression (FFT), resulting in fine-range accuracy.

We have also performed preliminary design simulations for our pulse-compression laser radar for a spaceborne altimeter application and have compared its performance with that of the ICESAT (Ice, Cloud and land Elevation Satellite) GLAS (Geoscience Laser Altimeter System) lidar. Our simulations show that a pulse-compression laser radar with an operating wavelength around $1310 \mathrm{~nm}, 1 \mathrm{~W}$ of peak power, $5 \mathrm{GHz}$ of transmitter bandwidth and a PRF of $4 \mathrm{kHz}$ could provide 200 elevation samples per second with an accuracy comparable to that of the GLAS lidar, which transmits $15 \mathrm{MW}$ peak power with a $40-\mathrm{Hz}$ PRF and obtains 40 elevation samples per second. The five-fold increase in elevation sample rate could then be used to provide elevation measurements across a swath, increasing the elevation mapping rate of the polar ice sheets.

Notable advances demonstrated in this proof-of-concept system include the ability to trade signal bandwidth for transmitter power in a laser radar while achieving a constant range accuracy, and the joining of modern radar processing techniques with fiber-optic technology in a laser radar. This is the first stage of a program to develop a near-infrared laser altimeter for spaceborne polar region monitoring.
\end{abstract}

\section{INTRODUCTION}

In July 2001, NASA plans to launch the Ice, Cloud and Land Elevation Satellite (ICESAT). Onboard this satellite will be the Geoscience Laser Altimeter System (GLAS), a spaceborne laser altimeter for measuring the surface topography of land, the ocean and the polar ice-sheets [1].
To achieve a $1-\sigma$ range accuracy of $10 \mathrm{~cm}$, the GLAS transmits 1064-nm wavelength, 15-MW peak power, 5-ns duration pulses at a pulse repetition frequency (PRF) of $40 \mathrm{~Hz}$ for an effective average power of $3 \mathrm{~W}$. High peak powers shorten laser life, and, in the case of GLAS, three lasers are required (two to meet the lifetime requirements and the third for redundancy). Further, for each pass of the satellite, the GLAS sensor will provide elevation samples based on the 70-m laser footprints at a $40 \mathrm{~Hz}$ rate yielding an effective along-track sample spacing of $173 \mathrm{~m}$ of the terrain below. This narrow swathwidth, coupled with the orbital track separation of about $7 \mathrm{~km}$ in southern Greenland, results in gaps in coverage that may be significant given our understanding of the spatial variability of the ice sheet dynamics.

Therefore a need exists for an altimeter that can provide accurate elevation data over a wide swath $(\geq 7 \mathrm{~km})$. This implies an increased pulse-repetition frequency (PRF) without significantly degrading the sensor lifetime or sacrificing accuracy. To fill this need, we are developing a laser radar altimeter that exploits advanced radar concepts that achieve good performance with low peak transmit power.

Instrument designs requiring lower peak powers may have advantages over conventional brute force systems in terms of complexity and efficiency. Holding the desired range accuracy constant, and recognizing how both the signal-tonoise ratio (SNR) and signal bandwidth (B) contribute to the overall range accuracy $\left(\sigma_{R}\right)$ through [2]

$$
\sigma_{\mathrm{R}}=\frac{\mathrm{c}}{2 \mathrm{~B} \sqrt{2 \mathrm{SNR}}},
$$

where $\mathrm{c}$ is the speed of light, we reason that through digital signal processing and appropriate design of the waveform and receiver, we can achieve comparable range accuracy with lower peak powers by applying modern radar techniques. Specifically, we can employ pulse compression and coherent integration to the design of the laser radar (altimeter) to trade peak power for bandwidth, pulse duration and observation time.

Applying these techniques with optical components was previously much more challenging than it is today, due to the development of key enabling technologies by the fiber-optic communications industry. Specific examples of fiber-optic components suitable for use in laser radars include singlemode solid-state lasers, low-loss single-mode fiber, photodetectors and modulators (both intensity and phase) with multi-GHz bandwidths, plus all the supporting hardware (connectors, couplers, splicing equipment, and test 
equipment). Especially significant is the development of the all-fiber-optic amplifiers such as the erbium-doped fiber amplifier (EDFA) with operating wavelengths around 1550 $\mathrm{nm}$ and the praseodymium-doped fluoride fiber amplifier (PDFFA) that operates around $1300 \mathrm{~nm}$. Yet more amplifiers with other operating wavelengths are in development. The availability of these technologies allow the development of efficient, compact laser radars that use modern radar concepts.

\section{SENSOR DESIGN}

To demonstrate the feasibility of a fiber-optic-based laser radar, we chose a simple chirp radar configuration that dechirps the received signal prior to digitization. The design is a hybrid of an RF radar system with an optical front end. Fig. 1 shows the block diagram of the laser radar with an emphasis on the optical components.

The low-power, continuous-wave $(\mathrm{CW})$ optical carrier signal produced in the laser diode is intensity modulated with the chirped RF signal that is generated in the RF radar system. The chirped optical signal is then amplified and routed through the multiplexer for transmission via free-space optics. We are exploiting a property of the EDFA that for a given gain (e.g., $30 \mathrm{~dB}$ ), for increasing input power signals, the output power is not limited in peak power (as is the case for most RF amplifiers), but rather the limitation is in terms of average power over the EDFA time constant $(\sim 10 \mathrm{~ms})$. During the receive operation, the backscattered optical signal is received via the same free-space optics, routed through the multiplexer to an optical amplifier, then filtered to a 1-nm spectral width prior to detection in the photodetector. The filtering rejects out-of-band optical power from background sources as well as amplified spontaneous emission (ASE) noise from the optical amplifier. The electronic output is sent to the microwave radar system to complete the signal processing.

In the RF part of the laser radar system (shown in Fig. 2), the chirp waveform is generated using direct digital synthesis (DDS). The timing and control signals (PRF and switch control) are derived from the same time base that drives the DDS. To reduce the requirements on the $\mathrm{A} / \mathrm{D}$ converter, the

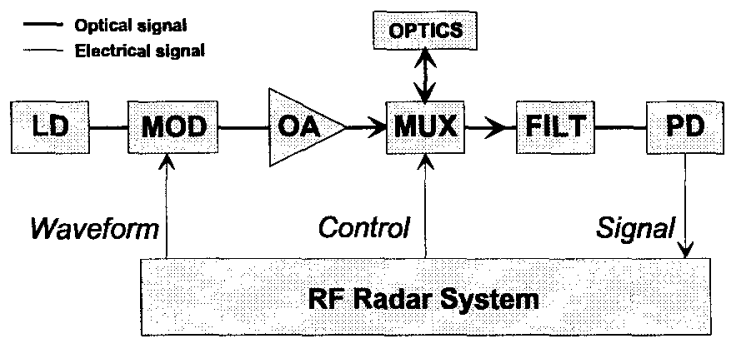

Figure 1. Functional block diagram of a laser radar using RF signal processing techniques. (LD: laser diode; OA: optical amplifier; MOD: modulator; MUX: optical multiplexer; FILT: bandpass filter; PD: photodetector.)

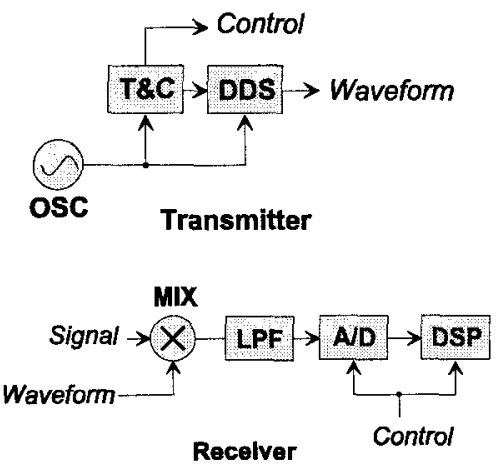

Figure 2. Functional block diagram of the transmitter and receiver portions of the microwave part of the laser radar. (OSC: RF oscillator; MIX: RF mixer; T\&C: timing and control subsystem; DDS: direct digital synthesizer; A/D: analog-to-digital converter; DSP: digital signal processor.)

signal is dechirped in the RF receiver prior to digitization with the effect of reducing the signal bandwidth; i.e., the bandwidth is proportional to the thickness of the sampled narrow range shell. For example, assuming a $10-\mu$ s pulse modulated with a $200-\mathrm{MHz}$ chirp (chirp rate of $20 \mathrm{MHz} / \mu \mathrm{s}$ ), and sampling a 50-m range shell, the bandwidth of the dechirped signal will be about $3.3 \mathrm{MHz}$. Therefore a digitization rate about $7 \mathrm{MHz}$ is sufficient, not the $400 \mathrm{MHz}$ rate that would have been required without dechirping.

For a system constrained to low peak transmit powers, the appropriate selection of the pulse duration, PRF, and number of coherent integrations will yield a significant improvement in SNR and, consequently, range accuracy, However this improvement alone may not be sufficient to provide a range accuracy comparable to a brute force system. For additional improvement we exploit the fact that $\sigma_{R}$ depends not only on SNR, but also on the bandwidth (B). Because of the development of high-performance fiber-optic components, it is possible to expand the bandwidth significantly from the requisite $200 \mathrm{MHz}$ (used in GLAS) to $5 \mathrm{GHz}$ (or more), with the effect of improving the range accuracy by a factor of 25 . To achieve a comparable improvement in $\sigma_{R}$ by improving the SNR would require an SNR improvement of $(25)^{2}$ or about $28 \mathrm{~dB}$.

\section{ANTICIPATED PERFORMANCE}

Applying this approach we have developed a baseline system design with the parameters shown in Table 1 . The anticipated range accuracy of this system is $10 \mathrm{~cm}$ (comparable to GLAS) with an output sample rate of 400 samples per second (10 times that of GLAS). This increased sample rate can translate into swath coverage with widths extending up to the desired $7 \mathrm{~km}$, depending on the required sample spacing. Assumptions used in this analysis include sensor altitude of $600 \mathrm{~km}$ with a ground-track speed of $6.9 \mathrm{~km} / \mathrm{s}$ and a sensor aperture diameter of $1 \mathrm{~m}$. To avoid the significant power penalty incurred in the photodetector due to 
its square-law characteristic, we assume an optically coherent receiver.

Wavelength dependence of the system performance (between 1 and $2 \mu \mathrm{m}$ ) is dominated by the spectral characteristics of the surface reflectance. Recognizing that the reflectance of ice and snow is weak at $1550 \mathrm{~nm}$, we plan to design our next-generation laser radar to operate in the $1310-\mathrm{nm}$ band where the reflectance is much closer to that seen at $1064 \mathrm{~nm}$. Sources, detectors, and modulators are also available at this wavelength; however all-fiber optical amplifiers at this wavelength are less common than at $1550 \mathrm{~nm}$. The availability of $1550-\mathrm{nm}$ optical amplifiers is why we selected this wavelength for our initial (proof-ofconcept) laser radar.

\section{TABLE 1. BASELINE SYSTEM PARAMETER COMPARISON}

$\begin{array}{ccc}\text { Parameters } & \text { Laser Radar } & \text { GLAS } \\ \text { Wavelength } & 1310 \mathrm{~nm} & 1064 \mathrm{~nm} \\ \text { Peak power } & 1 \mathrm{~W} & 15 \mathrm{MW} \\ \text { Pulse duration } & 25 \mu \mathrm{s} & 4 \mathrm{~ns} \\ \text { PRF } & 4 \mathrm{kHz} & 40 \mathrm{~Hz} \\ \text { Average power } & 100 \mathrm{~mW} & 2.4 \mathrm{~W} \\ \text { SNR per sample } & -50 \mathrm{~dB} & 13 \mathrm{~dB} \\ \text { Pulse compression gain } & 51 \mathrm{~dB} & 0 \mathrm{~dB} \\ \text { Signal processing gain } & 10 \mathrm{~dB} & 0 \mathrm{~dB} \\ \text { Final SNR } & 11 \mathrm{~dB} & 13 \mathrm{~dB} \\ \text { RMS range error } & 7.5 \mathrm{~cm} & 5-10 \mathrm{~cm} \\ \text { Along-track sample spacing } & 17 \mathrm{~m} & 173 \mathrm{~m}\end{array}$

\section{LABORATORY TEST RESULTS}

A proof-of-concept laser radar system was designed, assembled, and tested as a senior electrical engineering design project. Commercial RF-to-fiber-optic translators from Anacom were used to amplitude modulate and detect the $1550-\mathrm{nm}$ optical carrier. The RF chirp extends from 840 to $1000 \mathrm{MHz}$.

The RF transmitter subsystem forms the transmitted chirp that extends from 840 to $1000 \mathrm{MHz}$. An optical transmitter amplitude modulates a $1550-\mathrm{nm}$ wavelength laser with the RF chirp and directs it through a collimator toward the target. After reflecting off the target, a portion of the backscattered signal is collected in another collimator. The light is then detected (incoherent detection) and the RF chirp is recovered. This recovered chirp is then mixed with the transmitted chirp in the RF receiver. The resulting signal is a sinusoid with a frequency proportional to the target's distance. An analog-todigital converter (ADC) samples this waveform, followed by digital signal processing to determine the range to the target.

In basic laboratory testing we were limited to very short ranges $(<3 \mathrm{~m})$ due to the small receive aperture area of the collimator. For a specular target at a range of $2.68 \mathrm{~m}$, the

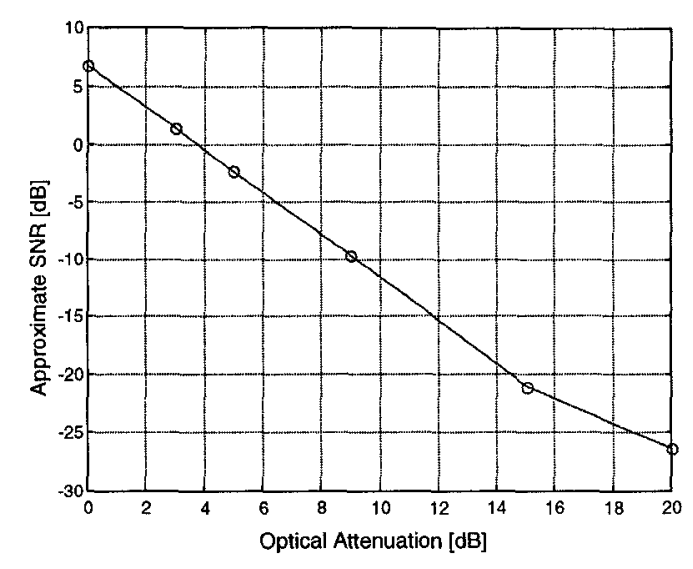

Figure3. Estimated SNR as a function of optical attenuation for which range estimates showed little or no accuracy degradation.

system measured a range of $2.74 \mathrm{~m}$, a difference of $6 \mathrm{~cm}$. We repeated this measure with decreased optical signal power (using an optical attenuator) and succeeded in extracting the signal from the noise through signal processing for SNRs as low as $-27 \mathrm{~dB}$, as shown in Fig. 3 .

\section{CONCLUSIONS}

We have demonstrated the concept of trading signal bandwidth for transmitter power in a laser radar system by applying pulse compression and coherent integration to achieve a good range accuracy despite a low peak transmitter power. In the future we plan to increase the system's operating range by improving the free-space interface with a telescope and improve the system sensitivity with optical amplifiers and optically coherent detection.

\section{ACKNOWLEDGMENTS}

The initial prototype of the laser radar with pulse compression was developed as part of a class project for electrical engineering seniors in the fall semester of 1998 at The University of Kansas. We wish to acknowledge the efforts of the students involved: Shane Haas, Carl Hunsinger, Samir Abdullah, Sarayuth Santaphongs, Lahsen Jguemouj, Kooksoon Loh, Sih Chung Lin, Yanki Cobanoglu, Andy Truong, and Sekken Chong.

\section{REFERENCES}

[1] Shutz, B.E., "Spaceborne laser altimetry: 2001 and beyond," in Book of Extended Abstracts, WEGENER98, H.P. Plab (Ed.), Norwegian Mapping Authority, Honefoss, Norway, 1998.

[2] Skolnik, M. I., Introduction to Radar Systems, McGrawHill, New York, 1980. 